\title{
RATE OF TERMINATION IN BULK POLYMERIZATION OF MMA INITIATED BY AIBN AND BPO*
}

\author{
NAOSHI OTOTAKE, FUMINORI UENO**, HIROMU TERADA*** \\ AND YUZO URAGUCHI \\ Department of Chemical Engineering, University of Tokyo, Tokyo
}

\section{Introduction}

Satisfactory treatments have never been made quantitatively on the acceleration of polymerization rate by radical mechanism. Some people have tried to relate the termination rate constant with viscosity of the reaction system. Though both viscosity and rate constant might be the function of both conversion and initiator concentration, it was difficult to correlate with each other directly.

In this paper, the apparent termination rate constant of the bulk polymerization of methylmethacrylate was measured. Then the rate constant was correlated with conversion and initiator concentration by an empirical equation. Further, a new dispersion model is proposed to extend the conversion range where the polymerization rate can be explained as the radicals are formed and destroyed at identical rates.

\section{Correlation of Apparent Termination Rate Constant with Experimental Data}

\section{1 Apparent rate constant, $\boldsymbol{k}_{i}{ }^{*}$}

Under the following assumptions, a set of well known equations have been derived. 1) Rate of radical formation $b y \dot{y}$ the thermal decomposition of initiator, $v_{i}$, and rate of radical disappearance, $v_{t}$, are in a state of balance. 2) Termination rate constant, $k_{t 0}$, initiator efficiency, $f$, decomposition rate constant, $k_{d}$, and propagation rate constant, $k_{p}$, are held constant. 3) Chain transfer reaction is neglected. 4) Termination occurs only by second order reaction of radical.

$$
\begin{aligned}
& v_{i}=2 f k_{d} c, \quad v_{p}=k_{p}\left(f k_{d} c / k_{t 0}\right)^{1 / 2}(M) \\
& v_{t}=2 k_{t 0}\left(R^{0}\right)^{2}, \quad v_{i}=v_{t}, \quad k_{t 0}=\text { const } \\
& -c_{0}{ }^{-1 / 2} \ln \left(1-x_{i}\right) \\
& =2 k_{p}\left(2 f / k_{t 0} k_{d}\right)^{1 / 2}\left[1-\exp \left(-k_{d} t / 2\right)\right] \\
& \vec{P}_{n t}=\int k_{p}\left(R^{*}\right)(M) d t / \int k_{t 0}\left(R^{*}\right)^{2} d t \\
& =M_{0} x_{i} / 2 f\left(c_{0}-c\right)
\end{aligned}
$$

If the assumption, $v_{i}=v_{t}$ is valid over the range where the acceleration results from the decrease of reation rate in the termination step alone, Eq. (4) is given corresponding to Eq. (2).

$$
\left.\begin{array}{l}
v_{i}=2 f k_{d} c, \quad v_{t}=2 k_{t}^{*}\left(R^{*}\right)^{2} \\
v_{p}=k_{p}\left(f k_{d} c / k_{t 0}\right)^{1 / 2}(M)\left(k_{t 0} / k_{t}^{*}\right)^{1 / 2}
\end{array}\right\}
$$

Eq. (5) is obtained corresponding to Eq. (3), where, $x$ is the observed conversion at reaction time $t$.

\footnotetext{
* Received on July 10, 1967

**: Sumitomo Shoji Co. Ltd:

*** Mitsubishi Rayon Co.
}

$$
\bar{P}_{n t}=M_{0} x / 2 f\left(c_{0}-c\right), \quad x=1-M / M_{0}
$$

Combination of Eqs. (2) and (4) gives Eq. (6)

$$
\mathrm{d} x / d x_{i}=(1-x) /\left(1-x_{i}\right) \cdot\left(k_{t 0} / k_{t}{ }^{*}\right)^{1 / 2}
$$

where, $x_{i}$ is the calculated conversion by Eq. (2) at the same reaction time. $k_{t}^{*}$ could be calculated, if $x$ and $\mathrm{d} x / \mathrm{d} x_{i}$ are observed and $x_{i}$ is calculated through known values of $f, c_{0}, k_{p}$ and $k_{t 0}$.

Here, $k_{t}^{*}$ is defined as an apparent termination rate constant.

\section{1-2 Measurement of conversion and correlation of rate con- stant with $c_{0}$ and $x$}

i) Contraction factor, $\alpha$ Bulk polymerization of MMA was carried out in a sealed glass tube using azo-bis-isobutyronitrile (AIBN) as an initiator. Experimental condition and constituents of monomer are shown in Table 1.

The volume of the tube was about $5 \mathrm{cc}$ and the shape of the tube was like a spheroidal so that heat could be removed easily. The temperature was regulated within $\pm 0.1^{\circ} \mathrm{C}$ around the desired value. The temperature rise at the center of the tube was found to be $3^{\circ} \mathrm{C}$ from the regulated temperature of the thermostat even at the conversion where acceleration occures conspicuously.

Dilatometry method was used to measure the conversion. In preparing the calibration curve, the conversion was determined by both gravimetric and double bond analysis.

The relation between $x$ and the volume contraction is shown in Fig. 1. Temperature dependence of $\alpha$ (total volume contraction equivalent to $100 \%$ conversion) is shown in Table 2.

\begin{tabular}{|c|c|c|c|c|c|}
\hline \multicolumn{4}{|c|}{ Experimental condition } & \multicolumn{2}{|c|}{ Constituents of monomer } \\
\hline$c:$ & $10^{-3}$ & $10^{-2}$ & $(\mathrm{~mol} / \mathrm{l})$ & water: & $0.002 \%$ \\
\hline$T:$ & 50 & 70 & $\left({ }^{\circ} \mathrm{C}\right)$ & free acid: & $0.007 \%$ \\
\hline$x:$ & 0 & 0.6 & $(-)$ & $\begin{array}{l}\text { inhibitor: } \\
\text { purity : }\end{array}$ & $\begin{array}{l}0.1 \text { ppm } \\
99.6 \%\end{array}$ \\
\hline
\end{tabular}

Though few reports which cover such wide range of $x$ have been presented, our result is in good agreement with that of Mackay et al. ${ }^{2)}$ in the range of lower conversion.

ii) Determination of $f$ As commercial monomer was used without further purification, induction period was determined first of all. Then the value of $f$ was deter-

Table 1. Experimental condition and constituents

of monomer used
Table 2
Dependence of $\boldsymbol{a}$ on temperature

$\begin{array}{cccc}T\left({ }^{\circ} \mathrm{C}\right) & 50 & 60 & 70 \\ \alpha & 0.315 & 0.32 & 0.33\end{array}$






Fig. I Relation between the observed conversion and the volume contraction at $60^{\circ} \mathrm{C}$.



Fig. 3 Comparison between Schulz's data and the conversion calculated by Eq. $(11-i)$

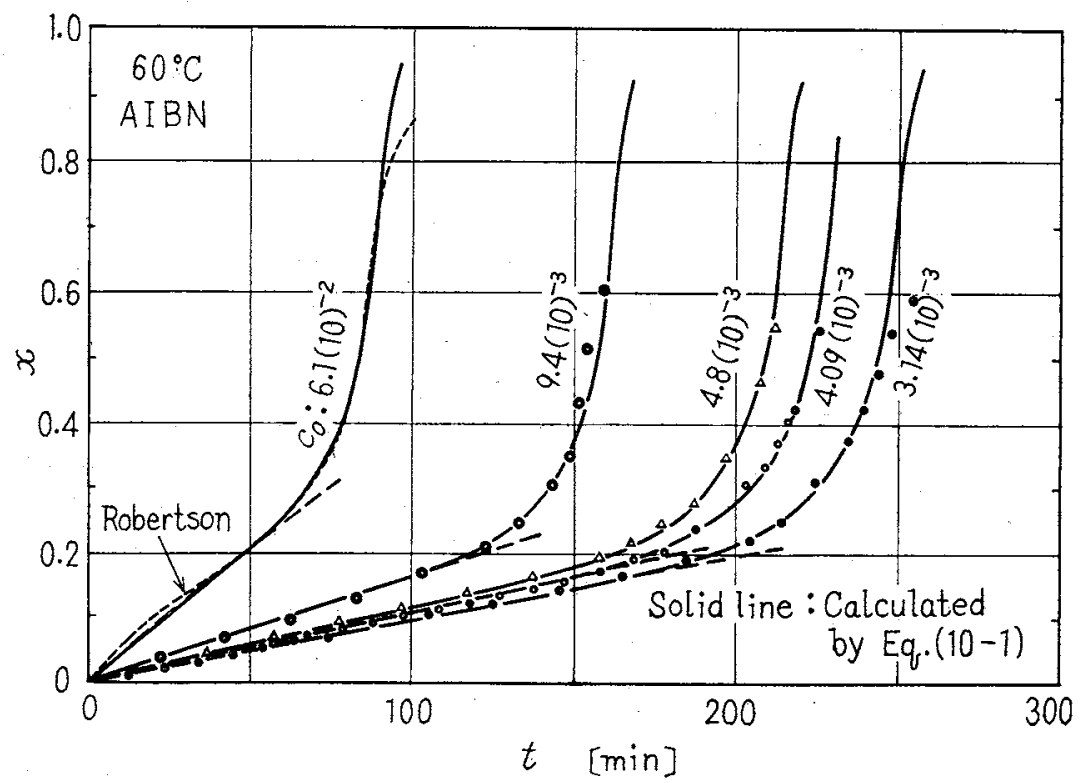

Fig. 2 Comparison beween the observed conversion and the calculated one by Eq. $(10-1)$

mined by trial and error method by using the following established rate constants for each elemental reaction, under the assumption that the value of $f$ depends only on the kind of initiator.

$$
\begin{aligned}
& k_{d}=1.18(10)^{14} \exp \left(-30(10)^{3} / R T\right), \quad \text { BPO } \\
& k_{d}=1.0(10)^{15} \exp \left(-30.7(10)^{3} / R T\right), \quad \text { AIBN } \\
& k_{p}=6.6(10)^{5} \exp \left(-4.7(10)^{3} / R T\right), \quad \text { MMA } \\
& k_{t 0}=4.9(10)^{9} \exp \left(-0.4(10)^{3} / R T\right), \quad \text { MMA }
\end{aligned}
$$

The value of $f$ was chosen to be 0.6 and 1.0 for AIBN and $\mathrm{BPO}$ respectively.

Dashed lines in Fig. 2 and Fig. $\mathbf{3}$ show the calculated conversion through Eq. (2) using rate constants of Eqs. (7) with the above determined values of $f$.

iii) Correlation of $k_{t}^{*} \quad$ Assuming that $k_{p}, k_{d}$ and $f$ are independent of $c_{0}, x$ and $t$, and further that over-all resistance of termination reaction is a sum of chemical resistance and diffusional resistance, the following relation is provided.
$1 / k_{t}^{*}=1 / k_{t 0}+1 / k_{t D}$

$k_{t}{ }^{*}$ was calculated from Eq. (6) using the observed conversion, $x$, and the value of $x_{i}$ calculated by Eq. (2) and Eq. (7). $k_{t 0} / k_{t D}=k_{t 0} / k_{t}{ }^{*}-1$, is supposed to be a function of polymer concentration (that is a function of $x$ ), average degree of polymerization, $\vec{P}_{n t}$, initiator concentration, $c$, temperature, $T$, and reaction time, $t$. In the case of constant temperature reaction, $x, c$ and $\bar{P}_{n t}$ depends only on $c_{0}$ and $t$, therefore $k_{t 0} / k_{t D}$ becomes a function of $c_{0}$ and $x$. Thus we can write,

$$
k_{t 0} / k_{t D}=F\left(c_{0}, x\right)
$$

Plotting $k_{t o} / k_{t D}$ so calculated vs. $x$, a group of straight lines is obtained with $c_{0}$ as a parameter as shown in Fig. 4. This suggests that function $F$ in Eq. $(9-1)$ can be written down in the form of $F\left(c_{0}, x\right)=F_{1}\left(c_{0}\right) x^{n}$, where dependence of $F$ on $c_{0}$ and $x$ is separated. Although the form of $F_{1}$ is not entirely clear, it is presumed to be directly proportional to $c_{0}{ }^{m}$ from the reason that the value of $k_{t 0} / k_{t D}$ at 
constant $x$ roughly fell on the straight line when it is plotted vs. $c_{0}$ on log-log paper. So that Eq. (9-1) can be converted to Eq. (9-2)

$$
\text { or, } \quad \begin{aligned}
k_{t 0} / k_{t D} & =K_{1}\left(c_{0}\right)^{m}(10 x)^{n} \\
k_{t}^{*} / k_{t s} & =1 /\left[1+K_{1}\left(c_{0}\right)^{m}(10 x)^{n}\right]
\end{aligned}
$$

For bulk polymerization of MMA using AIBN $(f=0.6)$ as an initiator, the following empirical equations were found to be appropriate.

$$
\begin{aligned}
& k_{t 0} / k_{t D}=2.3(10)^{-3}\left(c_{0}\right)^{-0.5}(10 x)^{6.8}, \text { at } 60^{\circ} \mathrm{C} \\
& k_{t o} / k_{t D}=2.0(10)^{-3}\left(c_{0}\right)^{-0.5}(10 x)^{5.0}, \text { at } 50^{\circ} \mathrm{C}
\end{aligned}
$$

Calculation of Eq. (10-1) are in good agreement with observation as shown in Fig. (2) for example.

The same procedure was applied to Schulz's data ${ }^{5)}$ carried out with $\mathrm{BPO}$ as the initiator and the similar cor-



Fig, 4 Correlation of $k_{t o} / k_{t D}$ with the observed conversion relations were obtained as follows.

$$
\begin{array}{ll}
k_{t 0} / k_{t D}=1.27(10)^{-4} c_{0}^{-0.74}(10 x)^{6.8}, & 70^{\circ} \mathrm{C} \\
k_{t 0} / k_{t D}=8.69(10)^{-3} c_{0}{ }^{-0.74}(10 x)^{5}, & 50^{\circ} \mathrm{C}
\end{array}
$$

In Fig. 3 are shown comparison between his observed data and calculation by Eq. (11-1).

Above discussion leads to the conclusion that Eq. (9-2) is a good approximation to apparent termination rate constant, where $m$ (signifies the contribution of $c_{0}$ ) is held almost constant regardless of temperature, but $n$ (signifies the contribution of $x$ ) is found to be effected by temperature.

Data reported by Robertson ${ }^{4)}$ and Trommsdroff ${ }^{57}$ are correlated by the same procedure.

\section{Extension of Stationary State Equation by New Dis- persion Model}

$2 \cdot 1$ Dispersion model

i) A concept Under the usual experimental codition, $\bar{P}_{n t}$ is in the order of $10^{3}$, which means polymers are much larger in size than a monomer molecule. If the propagating radicals are nearly the same in size with dead polymer, the reaction system is considered as a dispersed one. Propagating polymer radicals and formed polymers bind or adsorb monomers of certain volume per unit chain length. A mixture of polymer and bound monomer is defined as "a swollen polymer" and denoted SP hereinafter.

Authors supose four stages of mechanism for diffusion of polymer radicals.

1st stage : $x<x_{1}$. When the polymer concentration is low, the polymer fluctuates rather unrestrictedly through monomers as a SP. So that Eq. (2) may hold good.

2nd stage : $x_{1}<x<x_{2}$. As the reaction proceeds, volume fraction of SP reaches high enough to cause SPs to be bound one another and the polymer diffuses under the effect of entanglement. Concentration of segment in $\mathrm{SP}$ may be kept constant and the over all reaction proceeds according as unbound monomers decrease. Diffusivity of SP steeply decreases and accelerated reaction begins.

3 rd stage : $x_{2}<x<x_{3}$. If the reaction proceeds further, unbound monomer disappears and the whole reaction system apparently becomes a big swollen polymer. In this stage polymers or polymer radicals diffuse through SP. The concentration of segment in SP increases and bound monomers decrease with the increase in the extent of polymerization reaction.

4th stage: Perhaps the propagation constant varies sharply.

ii) Estimation of the presence of $\mathrm{SP}$ Precise data on

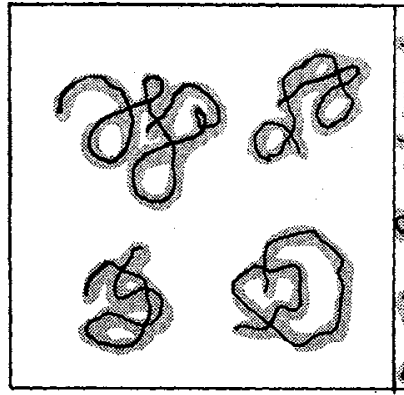

$x<x_{1}$

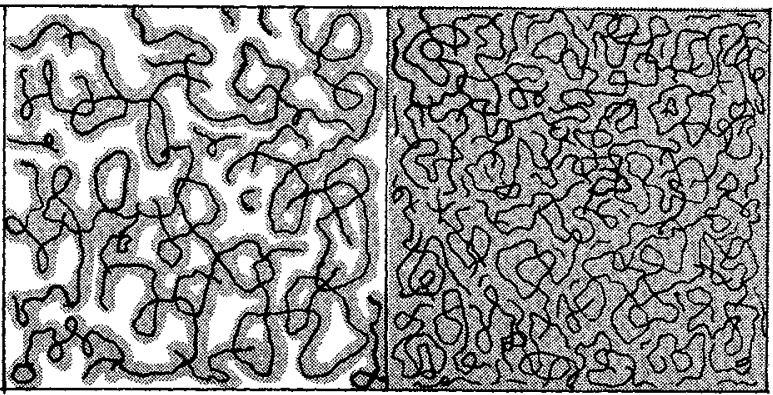

$x_{1}<x<x_{2}$ $x>x_{2}$
Fig. 5 Conceptional diagram for dispersion model 
the diffusion of MMA is not available. Fig. 6 shows the variation of diffusivity of benzene with polymer fraction where the diffusivity was calculated from the observed mutual diffusivity of rubber in benzene ${ }^{1}$. Solid line shows calculated value by applying Bruggeman and Rayleigh equation of electrical diffusivity ${ }^{3)}$ of benzene, where the diffusion in segment was neglected.

Above polymer concentration of $65 \%$, calcalation by Bruggeman's is in good agreement with observed data but apparent diffusivity of benzene in benzene is less than that of benzene in pure liquid benzene. In the range of below $65 \%$, observed diffusivity of benzene is in good agreement with calculation by Rayleigh's assuming that linear SP containing 35\% of benzene is dispersed in pure liquid benzene.

From the above consideration, it seems reasonable to estimate that polymer exists as a SP in the polymer concentration range below $65 \%$ and that the whole system becomes apparently a big SP mass in the concentration range of over $65 \%$. The same situation may be true to PMMA-MMA system.

\section{$2 \cdot 2$ Rate equation for 2 nd and 3 rd stage}

i) $\varphi_{p}, \varphi_{s}$ and $\alpha$, and their dependence on conversion and temperature From basic balances, the following equations are obtained;

$$
\left.\begin{array}{l}
n_{0}-n_{t}=n_{0} x, \quad V_{0}=n_{0} v_{M}, \quad N_{t} v_{s}=k\left(n_{0}-n_{t}\right) \\
\varphi_{s}=N_{t} v_{s} / V_{t}, \quad \alpha=\left(V_{0}-V_{t}\right) / V_{0} x
\end{array}\right\}
$$

where, $V$ is the volume of reaction system, $n$ the number of monomers, $N$, the number of segments in the system, $v_{M}$, the volume of unit monomer, $v_{s}$, the volume of unit segment and subscripts $o$ and $t$ refer to the condition at

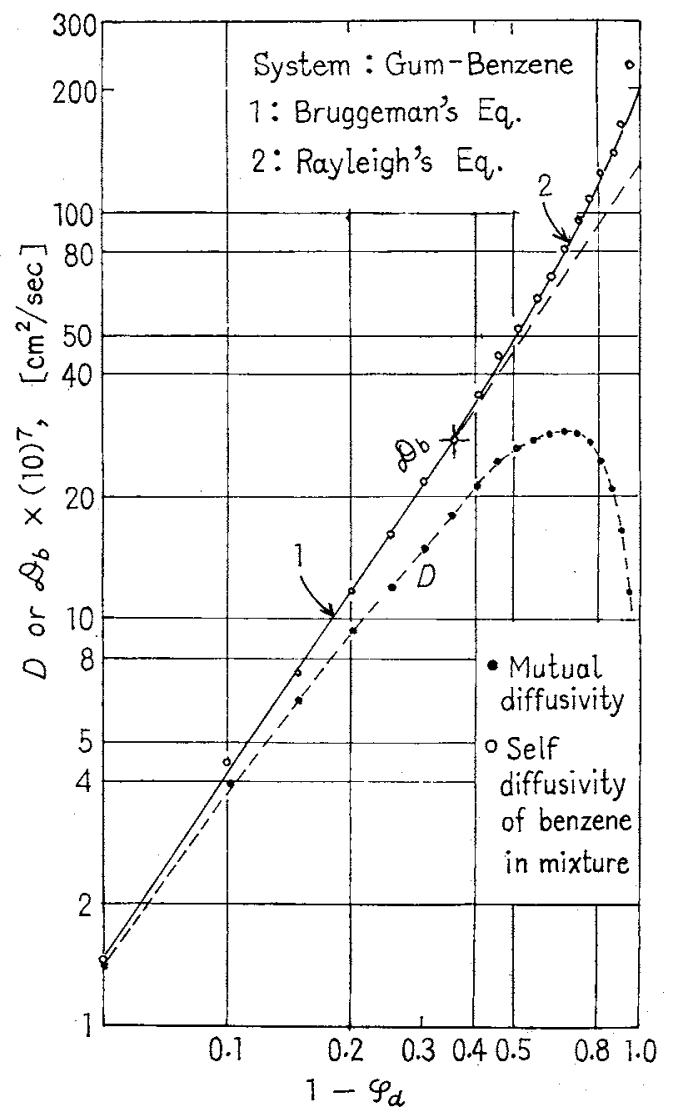

Fig. 6 The diffusivity of benzene in a gum-benzene mixture ${ }^{1)}$ $t=0$ and $t=t$, respectively, and then $\varphi_{p}, \varphi_{s}$ and $\alpha$ are the volume fraction of swollen polymer, that of segment and contraction factor in reaction system, respectively, and the following relation is derived.

$$
\begin{aligned}
\varphi_{s} & =k\left(n_{0}-n_{t}\right) / v_{M} n_{t}+k\left(n_{0}-n_{t}\right) \\
& =(1-\alpha) x /(1-\alpha x)
\end{aligned}
$$

Denoting the volume fraction of segment in SP to be $\varphi_{s p}$, the volume fraction of SP, $\varphi_{p}$, becomes

$$
\varphi_{p}=\varphi_{s} / \varphi_{s p}=\left(1-\alpha / \varphi_{s p}\right) x /(1-\alpha x)
$$

ii) Resistance against the diffusion of polymer radicals, $R_{D} \quad$ Generally speaking, diffusional resistance of particles suspended in the dispersed system is a function of the shape and the concentration of the particles concerned.

In the 2 nd stage, both polymers and radicals exist as a form of SP and fluctuate under the influence of other swollen polymers. The effect of interaction among SP is expected to be approximately proportional to $\varphi_{p}{ }^{2}$ and the resistance between entangled SPs at the constant concentration may be proportional to the square of intrinsic viscosity of SP which is proportional to $\bar{P}_{n t}{ }^{0.5} \sim \bar{P}_{n t}{ }^{0.6}$ according to Flory's theorem.

In the 3rd stage, un-bound monomers disappear and the whole reaction system apparently becomes a big swollen polymer where segments are dispersed in bound monomers. Therefore the diffusion of polymers or polymer radicals is supposed to be controled by the size of segment and the mutual hindering among segments. If the size of segments are constant, the resistance to the diffusion of segments will be proportional to the square of their concentration.

Thus, the following relations are provided.

$$
\begin{array}{ll}
R_{D} \propto \bar{P}_{n t}{ }^{1 / 1,2} \cdot \varphi_{p}{ }^{2} \\
\therefore \quad & R_{D} \propto \bar{P}_{n t} \cdot \varphi_{p}{ }^{2}, \quad \text { for } 2 \text { nd stage } \\
& R_{D} \propto \varphi_{s}{ }^{2}, \quad \text { for 3rd stage }
\end{array}
$$

$\varphi_{s p}$ is considered constant in 2 nd stage.

ii) Rate equation

$$
\begin{aligned}
& v_{p}=k_{p}\left(R^{*}\right)(M)=k_{p}\left(R^{*}\right)\left(M_{0}\right)(1-x) /(1-\alpha x) \\
& \text { if, } k_{t D} \ll k_{t 0}, \\
& \mathrm{~d} x / \mathrm{d} t=k_{p}\left(f k_{d} c / k_{t D}\right)^{1 / 2}(1-x) /(1-\alpha x)
\end{aligned}
$$

$k_{t D}$ in Eq. (8) may be inversely proportional to $R_{D}$. Substituting Eq. (14-1) into Eq. (15-1), we have Eq. (15-2) as the rate equation for 2 nd stage. Similarly substituting Eq. (14-2) into Eq. (15-1) we have Eq. (15-3) for 3rd stage. $\mathrm{d} x / \mathrm{d} t=K_{0}\left(c / c_{0}\right)^{1 / 2}\left(1-c / c_{0}\right)^{-1 / 2}(1-x) x^{3 / 2} /(1-\alpha x)^{2}$

$$
\mathrm{d} x / \mathrm{d} t=K_{1} c_{0}{ }^{1 / 2}\left(c / c_{0}\right)^{1 / 2} x(1-x) /(1-\alpha x)^{2}
$$

where, $K_{0}, K_{1}$, are constants determined by $k_{p}, k_{d}, \varphi_{s p}, \alpha$ and other proportional coefficients, and $c / c_{0}=e^{-k_{d} t}$ is the function of $t$.

\section{2-3 Verification of rate equation by experimental measure-} ments

i) Dependence of $x$ on $t$ and $c_{0}$ From Eq. (4), we have

$$
\mathrm{d} x_{i} / \mathrm{d} t=k_{p}\left(f k_{d} / k_{t 0}\right)^{1 / 2} c_{0}{ }^{1 / 2}\left(c / c_{0}\right)^{1 / 2}\left(1-x_{i}\right)
$$

Combining this equation with (15-2), the following expression is obtained for the same $c_{0}$ and $t$,

$$
\frac{(1-\alpha x)^{2}}{(1-x) x^{3 / 2}} \mathrm{~d} x \propto c_{0}^{-1 / 2}\left(1-c / c_{0}\right)^{-1 / 2} \cdot d\left(-\ln \left(1-x_{i}\right)\right)
$$

on the other hand, from Eq. (2) we have;

$$
c_{0}^{1 / 2}\left(1-\left(c / c_{0}\right)^{1 / 2}\right) \propto-\ln \left(1-x_{i}\right)
$$


therefore, $c / c_{0}$ becomes a function of $\ln \left(1-x_{i}\right)$ and the relation between $x$ and $\ln \left(1-x_{i}\right)$ can be obtained.

In the range of, $1>c / c_{0}>0.8,1-\left(c / c_{0}\right)^{1 / 2}$ is directly proportional to $1-c / c_{0}$. This approximation is nearly true for the reaction range reported by $\mathrm{Schul}$ where $\mathrm{BPO}$ was used as an initiator. Thus,

$$
\begin{aligned}
& \int \frac{(1-\alpha x)^{2}}{(1-x) x^{3 / 2} \mathrm{~d} x} \\
& \quad \propto c_{0}{ }^{-1 / 4} \int\left(-\ln \left(1-x_{i}\right)^{-1 / 2} \mathrm{~d}\left(-\ln \left(1-x_{i}\right)\right)\right. \\
& \quad 1>c / c_{0}>0.8
\end{aligned}
$$

As the function, $(1-\alpha x) / x^{3 / 2}$, is almost proportional to $x^{-2}$, the indefinite integral of the left hand side of Eq. (17-2) may be approximated by the following expression.

$$
\int \frac{(1-\alpha x)^{2}}{(1-x) x^{3 / 2}} \mathrm{~d} x \dot{\alpha} I_{0}-\left(\frac{1}{(1-\alpha) x}-\ln \frac{x}{1-x}\right)
$$

where, $I_{0}$ is an integral constant.

Fig. 7 shows the correlation of Schulz's data according to Eq. (17-3). It is obvious that in 2 nd stage, the linear relation is realized between them and that the critical conversions equivalent to $x_{1}, x_{2}$, and $x_{3}$ are distinctly recognized.

In the range of $x_{1}<x<x_{2}$, the slope of each straight line varies proportionally to $c_{0}^{-1 / 4}$, which precisely agrees with theoretical value, but in the range of $x_{2}<x<x_{3}$, they become nearly equal.

$x_{1}$ decreases with the decrease of $c_{0}$. This is considered reasonably by the fact that $\vec{P}_{n t}$ increases with decrease of $c_{0}$ at constant conversion. It is natural that $x_{2}$ is constant, because $x_{2}$ corresponds to the conversion where unbound monomers just disappear. Furthermore, it is interesting that $x_{3}$ is about 0.83 , which is equivalent to $\varphi_{s}=0.74$ in the case of $\alpha=0.32 .0 .74$ is very close to the value of the solid fraction in the closest packing of uniform spheres.

ii) Apparent termination constant, $k_{t}{ }^{*}$ Apparent termination constant is represented by Eq. (6). Combination of Eq. (15-1) with Eq. (15-2) and that of Eq. (15-1) with Eq. (15-3) give the following relations for 2 nd and $3 \mathrm{rd}$ stages.

$$
\begin{array}{ll}
k_{t 0} / k_{t}{ }^{*} \propto c_{0}{ }^{-1}\left(1-c / c_{0}\right)^{-1} x^{3} /(1-\alpha x)^{4} \\
k_{t 0} / k_{t}{ }^{*} \propto x^{2} /(1-\alpha x)^{4}, & \text { for } 2 \text { nd stage } \\
\end{array}
$$

and from the rate equations, are derived

$$
\begin{aligned}
&\left(1-c / c_{0}\right) \dot{\alpha}\left(\int \frac{(1-\alpha x)^{2}}{\left.(1-x) x^{3 / 2} \mathrm{~d} x+I_{0}\right)^{2},}\right. \\
& 1>c / c_{0}>0.8, \quad \text { for } 2 \text { nd stage } \\
& c_{0}{ }^{1 / 2}\left(1-c / c_{0}\right) \dot{\alpha}\left(\int \frac{(1-\alpha x)^{2}}{x(1-x)} \mathrm{d} x+I_{1}\right), \\
& 1>c / c_{0}>0.8, \quad \text { for 3rd stage }
\end{aligned}
$$

where $I_{0}, I_{1}$, are integral constants.

The right hand side of both equations are the only function of $x$ which means that the dependence of $k_{t 0} / k_{t}{ }^{*}$ on $c_{0}$ in 2 nd stage apparently varies from $c_{0}{ }^{-1}$ to $c_{0}{ }^{-1 / 2}$. Though the right hand side of Eq. (18-3) is affected by
Fig. 7 Correlation of Schulz's data according to Eq. (17)

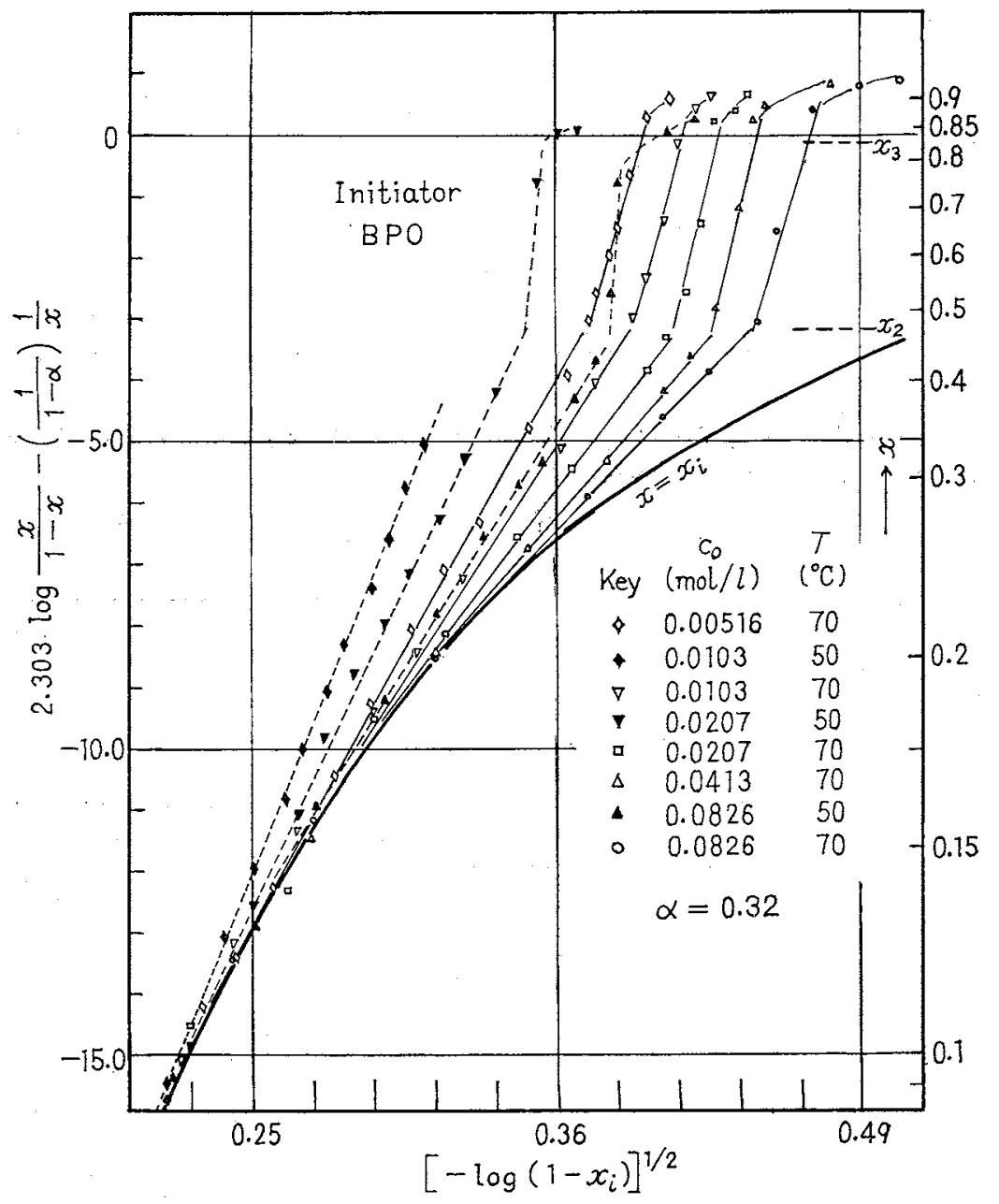


the value of $x_{1}$ and $x_{2}$, its numerical calculation decrease roughly with $x^{1.4} \sim x^{2.0}$.

Namely $k_{t 0} / k_{t}{ }^{*}$. varies with $x^{6} \sim x^{7.2}$. The results of these qualitative discussions agree with the empirical equation (11-1).

\section{Summary}

Under the assumption that $v_{i}=v_{t}$ is valid over the conversion range where the accelerated reaction occurs and that all the rate constants are kept constant except termination rate constant, the apparent termination rate constant, $k_{t}^{*}$, was correlated empirically with conversion, $x$, and initial concentration of initiator, $c_{0}$, by Eqs. $(9-2)$ and $(9-3)$. Such direct correlation of $k_{t}{ }^{*}$ with $c_{0}$ and $x$ was found to be better than that with viscosity of the reaction system.

Besides, authors proposed new dispersion model to explain the contribution of diffusion to polymerization reaction, where the mechanism of diffusion of polymer radicals in reaction system was divided into four stages. The rate equations, (15-2) and (15-3) were derived based on Eqs. (14) for 2 nd and 3 rd stages respectively.

Schulz's data were correlated and the empirical correlations obtained in Section 1 were also explained through the theoretical analysis.

\section{Acknowledgement}

The authors are indebted to the FUJIKURA KASEI Co. for financial support.

Nomenclature

$c, c_{0} \quad=$ initiator concentration at $t=t$ and $t=0$, respectively $[\mathrm{mol} / l]$

$$
\begin{aligned}
& f \quad=\text { initiator efficiency } \quad[-] \\
& I_{0}, I_{1} \quad=\text { integral constants } \\
& K_{0}, K_{1} \quad=\text { proportional coefficients } \\
& k_{d}=\text { thermo decomposition rate constant of initiator } \\
& k_{p}, k_{t 0}=\text { propagation and termination rate constant, } \\
& \text { repectively }[\mathrm{l} / \mathrm{mol} \cdot \mathrm{sec}] \\
& k_{t} * \quad=\text { apparent termination rate constant defined by } \\
& \text { Eq. (2) } \\
& \begin{array}{lll}
k_{t D} & =\text { defined by Eq. (8) } & {[l / \mathrm{mol} \cdot \mathrm{sec}]}
\end{array} \\
& M, M_{0} \quad=\text { monomer concentration at } t=t \text { and } t=0 \\
& \bar{P} \quad[\mathrm{~mol} / \mathrm{l}] \text { or }[\mathrm{g} / 100 \mathrm{ml}] \\
& \bar{P}_{n t} \quad=\text { number average degree of polymerization at } t=t \\
& \begin{array}{lll}
R & =\text { gas constant } \\
R^{*} & =\text { Concentration of radicals } & {[\mathrm{mol} / l]}
\end{array} \\
& R_{D} \quad=\text { diffusional resistance of propagating radicals } \\
& \text { SP } \quad=\text { "Swollen Polymer" defined in } 2-1 \\
& T \quad=\text { temperature } \\
& t \quad=\text { reaction time } \quad \text { [sec] or [min] } \\
& v_{i}, v_{p}, v_{t}=\text { radical initiation, polymer propagation and radical } \\
& \text { disappearance rate, respectively }[\mathrm{mol} / l \cdot \mathrm{sec}] \\
& x, x_{i}=\text { observed conversion and conversion calculated by } \\
& \text { Eq. (2) } \\
& \alpha=\text { contraction factor defined in } 1-2, \mathrm{i}) \quad[-] \\
& \varphi_{p}, \varphi_{s}, \varphi_{s p}=\text { concentration of swollen polymer, of segment and } \\
& \text { others }=k, N_{t}, n_{0}, n_{t}, V_{0}, V_{t}, v_{M} \text { and } v_{s} \text { are mentioned under }
\end{aligned}
$$

\section{Literature cited}

1) Hayes, M. and G. Park: Trans. Faraday Soc., 52, 949 (1956)

2) Mackay, M. and H. Melville: Trans. Faraday Soc., 45, 323 (1949)

3) Ototake, N.: Kagaku Kögaku, 31, 980(1967)

4) Robertson, E.: Trans. Faraday Soc., 52, 426 (1956)

5) Schulz, G. and G. Harborth: Makromol Chem., 1, 106 (1947)

6) Trommsdroff, E., E. Kohle and P. Lagally: Makromol Chem., 1, 169 (1948)

\section{FLUID AND PARTICLE DISPERSION IN FLUID-BED REACTORS ${ }^{*}$}

\section{-Experimental Investigations by Steady Heat-transfer and Steady Back-mixing of Adsorptive Gases-}

\section{Introduction}

The phenomenon of fluid mixing in fluid-bed reactors is related closely to the behaviour of the beds, particularly when stuch rate processes as heat- and mass-transfer and adsorption are superposed on catalytic reaction between

* Received on July 22, 1967

This work was presented before 31 st Annual Meeting of the Society of Chemical Engineers, Japan, at Tokyo, April 1966

** Central Research Lab., Kureha Chem. Co.

*** Central Research Lab., Asahi Glass Co.
TERUKATSU MIYAUCHI, HISATSUGU KAJI** AND KOJI SAITO*** Department of Chemical. Engineering, University of Tokyo, Tokyo reactant fluid and catalyst particles, and has been investigated by several workers ${ }^{2 \sim 5,9,12 \sim 17,20\rangle}$.

As for the mixing processes of bed particles, the works performed so far are rather confined to the topics on making clear the mechanisms of particle mixing or its fluid dynamic influence on fluid mixing, and little has been known about the influence of particle movement on the rate processes taking place in the beds. This means that the rate equations developed to describe the rate processes such as catalytic reaction or mass transfer usually do not include the influence of diffusive and convective 